\title{
INTENSIDADES DA PODA SECA E DO DESBASTE DE CACHO NA COMPOSIÇÃO DA UVA CABERNET SAUVIGNON
}

\author{
ALBERTO MIELE² \& LUIZ ANTENOR RIZZON ${ }^{3}$
}

RESUMO - A pesquisa foi realizada durante quatro anos, num vinhedo de Cabernet Sauvignon (Vitis vinifera L.), em Bento Gonçalves-RS. O objetivo foi determinar o efeito das intensidades da poda seca e do desbaste de cacho em variáveis relacionadas aos componentes de produção da videira e à composição do mosto de uva. Os tratamentos consistiram em dois níveis de poda seca - curta e longa - e quatro de desbaste de cacho (\%) - 0; 25; 50 e 75 -; com cinco repetições. O delineamento experimental foi o em blocos casualizados, com os tratamentos em parcelas subdivididas. Os resultados mostram que a poda seca e o desbaste de cacho tiveram efeito altamente significativo na produtividade do vinhedo que, na média dos quatro anos, variou de $10.971 \mathrm{~kg} / \mathrm{ha}$ - poda curta, $75 \%$ de desbaste - a $32.819 \mathrm{~kg} / \mathrm{ha}$ - poda longa, $0 \%$ de desbaste. Houve, também, efeito significativo na produtividade por gema, peso de ramos podados por gema e por hectare, área foliar/ peso fresco do fruto e produtividade/peso de ramos podados. Entretanto, o efeito nas variáveis relacionadas a açúcar e acidez do mosto da uva foi pouco expressivo. O componente 1 da análise de componentes principais discriminou o tratamento poda curta a $75 \%$ de desbaste de cacho dos tratamentos poda longa- $0 \%$ de desbaste e poda longa- $25 \%$ de desbaste.

Termos para indexação: manejo do dossel vegetativo, mosto, viticultura, Vitis vinifera.

\section{PRUNING AND CLUSTER THINNING INTENSITY ON THE COMPOSITION OF CABERNET SAUVIGNON GRAPE}

\begin{abstract}
The research was carried out in a Cabernet Sauvignon (Vitis vinifera L.) vineyard during four years in Bento Gonçalves-RS, Brazil. The objective was to determine the effect of the pruning and thinning intensity on the variables related to the grapevine yield components and grape must composition. Treatments consisted of two levels of pruning - short and long - and four levels of thinning (\%) - 0, 25, 50 and $75-$; there were five replicates. The experimental design was in a split-plot design. Results show that pruning and thinning had highly significant effect on the vineyard yield which varied from $10,971 \mathrm{~kg} /$ ha - short pruning- $75 \%$ cluster thinning - to $32,819 \mathrm{~kg} / \mathrm{ha}$ - long pruning- $0 \%$ cluster thinning - as average of four years. In addition, it was found significant effect on yield per bud, pruning weight per bud and per hectare, leaf area/fresh fruit weight and yield/pruning weight. However, there was a negligible effect on the variables related to sugar and acidity of the grape must. Component 1 of the principal component analysis discriminated short pruning- $75 \%$ cluster thinning from long pruning- $0 \%$ thinning and long pruning- $25 \%$ thinning.
\end{abstract}

Index terms: canopy management, must, viticulture, Vitis vinifera.

${ }^{1}$ (Trabalho 060-13). Recebido em: 28-01-2013. Aceito para publicação em: 12-11-2013.

2Engenheiro-Agrônomo, Dr., Embrapa Uva e Vinho, Caixa Postal 130, 95700-000 Bento Gonçalves-RS. E-mail: alberto.miele@ embrapa.br

${ }^{3}$ Engenheiro-Agrônomo, Dr., Embrapa Uva e Vinho, Aposentado. E-mail: luiz.rizzon@terra.com.br 


\section{INTRODUÇÃO}

O manejo do dossel vegetativo pode modificar a arquitetura da videira e ter efeito nos componentes de produção da planta, na composição do mosto da uva e na composição e nas características sensoriais do vinho. Entre as práticas utilizadas, destacam-se as intensidades da poda seca e do desbaste de cacho. Em geral, quando utilizadas, elas causam diminuição da produtividade do vinhedo e modificações nos processos bioquímicos e fisiológicos da planta e do fruto, os quais podem repercutir na qualidade da uva e do vinho.

A poda seca é uma prática realizada anualmente em regiões de clima temperado, com o principal objetivo de regular a produção temporal da videira (MANDELLI; MIELE, 2012). A definição da intensidade da poda pelo viticultor depende de vários fatores, mas, de toda maneira, procura-se harmonizar a produtividade do vinhedo com a qualidade da uva. Saliente-se que a produtividade do vinhedo correlaciona-se positivamente com o número de gemas deixadas por planta, ainda que, em geral, essa relação não seja linear.

O desbaste de cacho pode ser mais eficaz que a intensidade da poda na regulação da produção do vinhedo. Os resultados obtidos são variáveis, pois dependem, principalmente, da época em que é realizado e de sua intensidade. Além de diminuir o número de cachos por planta (REYNOLDS et al., 2007) e a produtividade do vinhedo (SUN et al., 2011), ele tem efeito em outros componentes de produção da videira, como a incidência da podridão cinzenta da uva, causada pelo fungo Botrytis cinerea (PALLIOTTI; CARTECHINI, 2000). Por outro lado, propicia aumento do peso do cacho e da baga (DAMI et al., 2006) e antecipa a maturação da uva (VALDÉS et al., 2009). Quanto a seu efeito sobre a composição do mosto de uva, em geral, o desbaste de cacho aumenta o ${ }^{\circ}$ Brix e o pH (DAMI et al., 2006), mas há trabalho mostrando que essa prática não tem efeito sobre essas variáveis (KELLER et al., 2005). Além disso, constata-se que ela também tem efeito no aumento da concentração de antocianinas e de taninos (PALLIOTTI; CARTECHINI, 2000), mas isso não é compartilhado por todos os autores (SUN et al., 2012). Normalmente, a qualidade da uva e do vinho está associada a produtividades moderadas ou baixas do vinhedo, enquanto as altas favorecem produtos de baixa qualidade.

Os principais açúcares da uva, glicose e frutose, são sintetizados na folha da videira pelo processo da fotossíntese, e sua concentração no fruto depende de fatores diversos. A área foliar, especialmente a das folhas expostas ao sol, é um importante fator para a produção desses açúcares e sua acumulação na uva. Assim sendo, a relação área foliar/peso fresco do fruto constitui-se em importante variável para determinar a qualidade da uva. Isso tem a ver com a capacidade da videira em sintetizar, transportar e acumular substâncias em quantidade suficiente para maturar adequadamente o fruto (KLIEWER; WEAVER, 1971). Os parâmetros da relação área foliar/peso fresco do fruto, indicados para a produção de uva de qualidade, variam de acordo com as características de cada vinhedo e de sua localização. Dessa maneira, para a produção de vinho Merlot de qualidade, foram necessários 15 $\mathrm{cm}^{2}$ de folha/g de fruto (BUBOLA et al., 2011) e constatou-se aumento considerável do ${ }^{\circ}$ Brix quando essa relação passou de 6 a $12 \mathrm{~cm}^{2}$ de folha/g de fruto (PASTORE et al., 2011).

A relação produtividade do vinhedo/peso de ramos podados também é utilizada para estabelecer parâmetros que definam a qualidade da uva, pois ela se relaciona ao equilíbrio entre as partes produtiva e vegetativa da planta. Os valores que melhor expressam essa relação variam em função de um conjunto de fatores, devendo estar compreendidos entre 4,6 e 12,0 (BRAVDO et al., 1985).

A escolha de práticas culturais visando a modificar o dossel vegetativo do vinhedo e a composição da uva, depende basicamente das condições edafoclimáticas de cada região. Por isso, este trabalho teve o objetivo de determinar o efeito de diferentes níveis de poda seca e de desbaste de cacho nos componentes de produção da videira, na produtividade do vinhedo e na composição do mosto da uva Cabernet Sauvignon cultivada na Serra Gaúcha.

\section{MATERIAL E MÉTODOS}

O experimento foi realizado durante quatro ciclos vegetativos da videira, num vinhedo formado com o cv. Cabernet Sauvignon localizado na Embrapa Uva e Vinho, em Bento Gonçalves-RS.

A formação do vinhedo foi feita por meio do plantio de estacas do porta-enxerto SO4 (Vitis berlandieri Planch. $\mathrm{x}$ Vitis riparia Mchx.), em covas previamente abertas e preparadas na área do experimento. O plantio foi realizado colocando-se uma estaca do porta-enxerto em cada cova. Nos dois anos seguintes, foi necessário estaqueá-los e amarrar os ramos. A enxertia, de garfo, foi realizada a campo durante o inverno, quando eles tinham dois anos. Para isso, utilizou-se material vegetativo do cv. Cabernet Sauvignon procedente de um vinhedo 
produtivo e visualmente isento de doenças fúngicas e de viroses, de modo similar à seleção do material vegetativo dos porta-enxertos.

As videiras foram conduzidas em latada, estando o aramado a uma altura de $1,85 \mathrm{~m}$ do solo. $\mathrm{O}$ espaçamento entre as fileiras foi de $2,5 \mathrm{~m}$, e entre as plantas, nas fileiras, de 1,8 m. Portanto, a densidade de plantio foi de 2.222 plantas/ha. Adotou-se a poda mista, deixando-se uma média de 6 varas/planta e 10 esporões com duas gemas/planta.

Durante os quatro ciclos vegetativos da videira, controlaram-se as doenças fúngicas com pulverizações de fungicidas específicos para cada agente causal. Contudo, não foram feitas pulverizações e/ou polvilhamentos de inseticidas, bactericidas e reguladores de crescimento nem aplicação de corretivos da acidez do solo e de fertilizantes.

Os tratamentos consistiram em dois níveis de poda seca e em quatro de desbaste de cacho. Os níveis de poda foram poda curta (PC), onde as varas foram podadas e amarradas no primeiro fio, situado a $0,40 \mathrm{~m}$ do fio de condução da planta e poda longa (PL), onde as varas foram podadas e amarradas no segundo fio, situado a $0,70 \mathrm{~m}$ do fio de condução da planta. O número total de gemas/ha foi determinado em função do tipo de poda e do vigor das plantas. Assim, nos tratamentos de PC, deixou-se, do primeiro ao quarto ano, respectivamente, o equivalente a 138,0 mil, 167,6 mil, 140,8 mil e 177,5 mil gemas/ha; nos tratamentos de PL, 217,5 mil, 241,6 mil, 193,1 mil e $232,2 \mathrm{mil}$ gemas/ha, respectivamente. Isso significa que, na média dos quatro anos, o conjunto dos tratamentos de PL teve $41,8 \%$ de gemas a mais que os da PC. As operações de poda seca nas videiras foram realizadas no terceiro decêndio de agosto de cada ano. Os tratamentos de poda seca e de desbaste de cacho foram sempre realizados nas mesmas videiras, a fim de se constatar o efeito cumulativo desses tratamentos.

Os níveis de desbaste de cacho foram $0 \%$ (0D), 25\% (25D), 50\% (50D) e 75\% (75D), os quais foram efetuados no pegamento do fruto. $\mathrm{O}$ desbaste de cacho foi feito, sempre que possível, de forma alternada na vara e no esporão. Além disso, desbastaram-se, sucessivamente, os cachos de acordo com a posição que eles estavam inseridos nas varas e nos esporões de cada planta, isto é, cacho 1 da vara 1 , cacho 1 do esporão 1 ; cacho 2 da vara 2 , cacho 2 do esporão 2; cacho $n$ da vara $n$, cacho $n$ do esporão $n$. O desbaste foi realizado durante o terceiro decêndio de novembro de cada ano. À exceção do desbaste de cacho, não foi realizado nenhum outro tipo de poda verde, como desfolha, desponta, desbrota, desnetamento e esladroamento.

Portanto, houve oito tratamentos-dois níveis de poda seca e quatro de desbaste de cacho-dispostos em blocos ao acaso, e cinco repetições. Os níveis de poda formaram as parcelas e os de desbaste de cacho, as subparcelas, as quais foram constituídas por 10 plantas, com área de $45 \mathrm{~m}^{2}$ cada uma delas. Assim, o experimento foi formado por 400 plantas que ocuparam uma área de $1.800 \mathrm{~m}^{2}$.

Por ocasião da maturação da uva, constatada por meio de um refratômetro manual, procedeuse à colheita. A uva de cada parcela foi colocada em caixas de plástico com capacidade de $20 \mathrm{~kg}$, pesada, desengaçada e esmagada. Após, coletaramse amostras de $170 \mathrm{~mL}$ de mosto, as quais foram centrifugadas antes de serem analisadas.

As variáveis avaliadas na videira foram: a) produtividade da gema (g/gema), obtida dividindose o peso de uva pelo número total de gemas; b) produtividade do vinhedo $(\mathrm{kg} / \mathrm{ha})$, que é igual ao peso da uva produzida/ha; c) peso de ramos podados/ gema (g/gema), representado pelo peso dos ramos podados no inverno dividido pelo número total de gemas; d) peso de ramos podados/ha, que é igual ao peso dos ramos podados/ha; e) relação área foliar/ peso fresco do fruto $\left(\mathrm{cm}^{2} / \mathrm{g}\right)$, obtida dividindo-se a área foliar pelo peso fresco da uva; e f) relação produtividade do vinhedo/peso de ramos podados $(\mathrm{kg} / \mathrm{kg})$, dada pela divisão da produtividade do vinhedo pelo peso dos ramos podados. A área foliar foi determinada com um medidor de área foliar de bancada, marca LI-COR, fazendo-se mensurações de folhas previamente amostradas. Determinados a área média das folhas e o número de folhas de cada tratamento, calculou-se a área foliar das subparcelas através de uma relação simples.

As variáveis avaliadas no mosto da uva foram ${ }^{\circ}$ Brix, densidade, acidez titulável e $\mathrm{pH}$. O ${ }^{\circ}$ Brix foi determinado com um refratômetro com correção de temperatura, tipo Abbe, da American Optical Corporation; a densidade, com um densímetro digital com correção de temperatura, marca PAAR; a acidez total, por titulação; e o pH, com pHmetro Corning (RIBÉREAU-GAYON et al., 1982).

Os dados obtidos foram submetidos à análise de variância e ao teste de Tukey, segundo o modelo correspondente, e à análise de componentes principais.

\section{RESULTADOS E DISCUSSÃO}

As intensidades da poda seca e do desbaste de cacho causaram efeito altamente significativo $(\mathrm{p}<0,001)$ na produtividade/gema nos quatro ciclos 
vegetativos da videira (Tabela 1). Constata-se que os tratamentos mais intensos - PC-75D, PL75D, PC-50D e PL-50D - estiveram entre os que proporcionaram produtividades mais baixas em todos os anos. De fato, as médias desses tratamentos, nos quatro anos avaliados, foram, respectivamente, de 72,$8 ; 80,3 ; 106,8$ e 111,0 g/gema. No que se relaciona ao desbaste de cacho, dentro de poda seca, a média da produtividade/gema (g/gema) nos quatro anos foi de 154,5 (0D), 130,2 (25D), 108,9 (50D) e 76,6 (75D). Portanto, as diferenças em relação ao tratamento 0D foram de $-15,7 \%$ (25D), $-29,5 \%$ (50D) e $-50,4 \%$ (75D). Mas a produtividade/gema nos dois tratamentos de poda, dentro de desbaste de cacho, foi similar, ou seja, 117,6 g/gema em PC e 117,4 g/ gema em PL. Isso evidencia que o desbaste de cacho foi mais eficaz que o nível de poda na diminuição da produtividade/gema.

O resultado da produtividade/gema repercutiu diretamente na produtividade do vinhedo, pois esses tratamentos, em geral, também tiveram as produtividades mais baixas (Tabela 1). Nesse período, a amplitude da produtividade média nos quatro anos foi de $21.847,7 \mathrm{~kg} / \mathrm{ha}$, variando de $32.818,5 \mathrm{~kg} / \mathrm{ha}$ no tratamento com PL-0D (menos intenso) a 10.970,8 kg/ha no tratamento com PC-75D (mais intenso); houve, portanto, uma produtividade 2,99 vezes maior no tratamento com PL-0D. Já o tratamento com PC-0D, o mais adotado na Serra Gaúcha, teve uma média de $22.841,8 \mathrm{~kg} / \mathrm{ha}$, ou seja, 30,4\% menor que a do tratamento com PL-0D. Comparando as produtividades dos tratamentos com PL e PC, verifica-se que elas variaram de $25.522,8$ $\mathrm{kg} /$ ha a $17.775,1 \mathrm{~kg} / \mathrm{ha}(-30,4 \%)$, respectivamente. No que se relaciona ao desbaste de cacho, as produtividades dos vinhedos $(\mathrm{kg} / \mathrm{ha})$ foram $27.830,2$ (0D), 23.864,3 (25D), 20.687,4 (50D) e 14.213,9 (75D). As diferenças de produtividade em relação ao tratamento 0D foram -14,3\% (25D), -25,7\% (50D) e $-48,9 \%$ (75D). Portanto, o desbaste de cacho 75D foi mais eficaz em diminuir a produtividade do vinhedo que a poda curta, pois houve uma diferença de 18,5 pontos porcentuais entre eles.

Os tratamentos com poda longa tiveram maior número de gemas/planta que os de poda curta, o que, por si só, proporcionou maior número de gemas por unidade de área. Isso implica maior número de cachos e, consequentemente, maior produtividade do vinhedo (MURISIER; ZIEGLER, 1991). Por outro lado, o desbaste de cacho reduz o número de cachos/ planta e por unidade de área (REYNOLDS et al., 2007), induzindo a menor produtividade do vinhedo (KELLER et al., 2005; DAMI et al., 2006; KELLER et al., 2008; GIL-MUNÕZ et al., 2009; VALDÉS et al., 2009; BERKEY et al., 2011; BUBOLA et al., 2011; KOK, 2011; SUN et al., 2012).

A poda e o desbaste de cacho tiveram efeito significativo $(\mathrm{p}<0,001)$ no peso de ramos podados/ gema (Tabela 2) nos quatro anos avaliados. Os tratamentos com PC foram os que, em geral, proporcionaram maiores pesos de ramos podados, o que era esperado, pois nesses tratamentos retiraramse maiores quantidades de ramos que nos com PL. Entretanto, nos dois últimos anos de PL-50D e nos três últimos de PL-75D, esses pesos também se situaram entre os mais elevados. Constata-se, ainda, que em todos os anos o peso de ramos podados foi maior nos tratamentos com PC, sendo 71,1\% maior na média dos quatro anos. Isso pode estar relacionado ao aumento de vigor do ramo durante seu crescimento. Este fato tem a ver com a maior relação fonte/dreno metabólico e com a repartição de assimilados.

No que se relaciona ao peso de ramos podados/ha (Tabela 2 ), houve diferença significativa somente no $1^{\circ}$ ano $(\mathrm{p}<0,01)$, mas os resultados foram inconsistentes. Comparando-se os tratamentos com PC e PL deste ano, constata-se que a PC teve peso de ramos podados $22,6 \%$ maior que a PL. Isso ocorreu porque, nos tratamentos com PC, houve maior quantidade de material podado. Quanto ao desbaste de cacho, verifica-se que quanto mais intensa for esta prática, maior será o peso de ramos podados (EZZAHOUANI; WILLIAMS, 2001 DAMI et al., 2006; SUN et al., 2012). Isso significa que a translocação dos fotossintetizados para os cachos, que se constituem em importantes drenos metabólicos, foi em detrimento do crescimento dos ramos. Entretanto, há resultado de pesquisa que mostra que não houve efeito do desbaste de cacho no peso de ramos podados (KELLER et al., 2005).

$\mathrm{O}$ efeito da poda e do desbaste de cacho foi altamente significativo $(\mathrm{p}<0,001)$ na relação área foliar/peso fresco do fruto (Tabela 3 ) dos quatro anos avaliados. Em geral, os tratamentos de PC-75D e de PL-75D apresentaram os valores mais elevados. De fato, essa relação foi, em média, de $47,90 \mathrm{~cm}^{2}$ de folha/g de fruto fresco no tratamento de PC-75D e de $38,06 \mathrm{~cm}^{2}$ de folha/g de fruto fresco no de PL75D. Comparando os tratamentos de PC e PL, para os quatro níveis de desbaste de cacho, constata-se que aquele foi 22,8\% superior a este. Quanto ao desbaste de cacho, dentro de poda, a relação área foliar/peso fresco do fruto do tratamento 75D com os demais foi, na média dos quatro anos, de 116,1\% (0D), 92,6\% (25D) e 54,0\% (50D) mais elevada. As maiores relações área foliar/peso fresco do fruto dos tratamentos de PC-75D e PL-75D foram 
devidas, especialmente, às menores produtividades proporcionadas por esses tratamentos. Portanto, o desbaste de cacho aumentou a relação área foliar/ peso fresco do fruto, o que está de acordo com resultados de outros autores (BUBOLA et al., 2011; PASTORE et al., 2011). Saliente-se, também, que a relação área foliar/peso fresco do fruto correlacionouse negativamente com a produtividade do vinhedo. Os valores variaram de $9,08 \mathrm{~cm}^{2} / \mathrm{g}$ no tratamento com PL-0D no $1^{\circ}$ ano, a $64,44 \mathrm{~cm}^{2} / \mathrm{g}$ no de PC$75 \mathrm{D}$, no $4^{\circ}$ ano. $\mathrm{O}$ tratamento de $\mathrm{PC}-0 \mathrm{D}$, que pode ser considerado como o referencial adotado pelos viticultores na Serra Gaúcha, variou de $16,70 \mathrm{~cm}^{2} / \mathrm{g}$ no $3^{\circ}$ ano a $26,93 \mathrm{~cm}^{2} / \mathrm{g}$ no $4^{\circ}$ (Tabela 3 ).

Essa relação é importante para determinar a concentração desejada de açúcar no mosto, estimar a qualidade do vinho e, ainda, dá uma indicação do desbaste a ser feito, pois está relacionada com a capacidade da videira em armazenar carboidratos. Esses valores estão, em geral, acima dos considerados como recomendáveis para a videira, ou seja, de 7 a $10 \mathrm{~cm}^{2} / \mathrm{g}$ (KLIEWER; ANTCLIFF, 1970; SMART, 1985). Valores superiores a $10 \mathrm{~cm}^{2} / \mathrm{g}$ em Cabernet Sauvignon demonstraram ter menos efeito sobre o teor de açúcar do mosto (JACKSON, 1986), e abaixo de $8 \mathrm{~cm}^{2} / \mathrm{g}$, o crescimento vegetativo fica comprometido (KAPS; CAHOON, 1992). Entretanto, há resultado de pesquisa que indica que valores abaixo de $18 \mathrm{~cm}^{2} / \mathrm{g}$ reduzem a qualidade do vinho (NAOR et al., 2002). Isso significa que há uma série de fatores que podem afetar o efeito da relação área foliar/peso fresco do fruto. Considerese, entretanto, que os parâmetros referenciados relacionam-se a vinhedos conduzidos em espaldeira, de produtividades relativamente baixas e cultivados em regiões vitícolas com terroirs distintos dos da Serra Gaúcha. No caso de vinhedos conduzidos em latada, essa relação pode ser superior, a qual ainda necessita ser estabelecida.

No que diz respeito à relação produtividade/ peso de ramos podados, houve efeito altamente significativo $(\mathrm{p}<0,001)$ nos quatro anos (Tabela 3). Em geral, os tratamentos de PL-0D e PL-25D apresentaram os maiores valores dessa relação, os quais foram, na média dos quatro anos, 7,89 e 6,57, respectivamente. Na comparação dos tratamentos entre PC e PL, na média dos quatro anos, este último foi $75,7 \%$ maior. No que se relaciona ao desbaste de cacho, dentro de poda, a relação produtividade/ peso de ramos podados do tratamento $0 \mathrm{D}$ em relação aos demais foi, na média dos quatro anos, $152,7 \%$ (75D), $74,1 \%$ (50) e $14,1 \%$ (25D) mais elevada. Esses resultados evidenciam a forte influência do desbaste de cacho na relação produtividade/peso de ramos podados, a qual diminui proporcionalmente à intensidade do desbaste de cacho (KELLER et al., 2005; DAMI et al., 2006; KELLER et al., 2008; SUN et al., 2012). Da mesma forma que a relação área foliar/peso fresco do fruto, a relação produtividade/ peso de ramos podados também é usada como referência para a qualidade do produto (BRAVDO et al., 1984), sendo negativamente correlacionada àquela. Os parâmetros da relação produtividade/ peso de ramos podados deste trabalho estão, em geral, aquém dos mencionados na literatura como valores indicados para a produção de uva destinada a vinhos de qualidade, ou seja, situados numa faixa que varia de 4,6 a 12,0, pois valores inferiores a 4,6 não favorecem a melhoria da qualidade do vinho, e superiores a 12,0 a diminuem (BRAVDO et al., 1985). Isso deveu-se à poda curta e a plantas vigorosas, as quais proporcionaram crescimento e desenvolvimento exuberante dos ramos. Embora essa relação seja mencionada como uma valiosa ferramenta, ela, por si só, apresenta alguns problemas, como, por exemplo, o dossel vegetativo pode ter variação no número de folhas expostas ao sol, o qual pode modificar a área foliar efetiva por unidade de peso do fruto (JACKSON; LOMBARD, 1993). Além disso, tem-se que considerar as características de solo e o clima de cada região e das práticas culturais postas em prática nos vinhedos.

O efeito dos níveis de poda seca e de desbaste de cacho nas variáveis relacionadas ao mosto da uva Cabernet Sauvignon (Tabelas 4 e 5) não foi tão expressivo quanto aquele das variáveis relacionadas aos componentes de produção da videira. De fato, a poda e o desbaste de cacho não tiveram efeito significativo no ${ }^{\circ}$ Brix (Tabela 4 ), em três anos avaliados. No $2^{\circ}$ ano, o tratamento com PL-75D $(23.375 \mathrm{~kg} / \mathrm{ha})$ teve ${ }^{\circ}$ Brix maior $(\mathrm{p}<0,05)$ que o com PL-0D (38.689 kg/ha), ou seja, foi necessária uma diminuição da produção de uva de 15.314 $\mathrm{kg} / \mathrm{ha}$ para se obter uma elevação de $0,8{ }^{\circ}$ Brix. Entretanto, no conjunto dos quatro anos, variações de produtividades médias do vinhedo, da ordem de $10.970,8 \mathrm{~kg} / \mathrm{ha}$ (PC-75D) e 32.818,5 kg/ha (PL-0D), não proporcionaram considerável elevação no ${ }^{\circ}$ Brix do mosto da uva Cabernet Sauvignon. A densidade (Tabela 4), como se esperava, teve comportamento similar ao do ${ }^{\circ}$ Brix, pois houve diferença significativa entre os tratamentos, em dois anos $(\mathrm{p}<0,05)$, em que, no $1^{\circ}$ ano, PC-75D diferenciou-se de PL-0D e, no $2^{\circ}$, foram PC-0D e PL-75D que se diferenciaram de PL-0D. A acidez titulável (Tabela 5) foi significativa $(\mathrm{p}<0,05)$ somente no $1^{\circ}$ ano, e o pH (Tabela 5) só não o foi no $3^{\circ}$ ano. Em ambos os casos, os resultados não foram consistentes. 
Com o intuito de discriminar os tratamentos e de fornecer subsídios para melhor compreensão do efeito de dois níveis de poda seca e de quatro de desbaste de cacho nos componentes de produção da videira e na composição físico-química do mosto da uva Cabernet Sauvignon, submeteram-se os dados das médias dos quatro anos de avaliação à análise de componentes principais (ACP). Isto porque a ACP é uma ferramenta extremamente útil para discriminar tratamentos de determinados experimentos onde o teste de comparação de médias se mostra, de certa forma, pouco discriminatório. Neste trabalho, os componentes principais 1 e 2 representaram $67,30 \%$ e $16,51 \%$, respectivamente, da variação total (Figura $1 \mathrm{~A}, \mathrm{~B})$.

O componente principal 1 (CP 1) (Figura 1A) discriminou o tratamento com PC-75D de PL-0D e PL-25D e o 2 (CP 2), o PC-50D do PL-75D. O CP 1 - entre parênteses, estão os coeficientes de correlação entre as variáveis e os componentes principais caracterizou-se pelas variáveis produtividade/peso de ramos podados (PPR) $(0,98)$, produtividade/ ha (PHA) $(0,97), \mathrm{pH}(\mathrm{PH})(-0,95)$, peso de ramos podados/ha $(\mathrm{PRH})(-0,88)$, área foliar/peso fresco do fruto (APF) $(-0,87)$, produtividade/gema (PGE) $(0,77),{ }^{\circ}$ Brix $(\mathrm{BRX})(-0,76)$, peso de ramos podados/ gema (PRG) $(-0,75)$ e densidade (DEN) $(-0,70)$; no CP 2, o tratamento com PC-50D pela acidez titulável (ACT) (-0,77). Isso significa que, com PC$75 \mathrm{D}$, o mais intenso dos tratamentos - e de menor produtividade - caracterizou-se (Figura 1B) por valores mais elevados de ${ }^{\circ}$ Brix, densidade, $\mathrm{pH}$, peso de ramos podados/gema, peso de ramos podados/ ha e área foliar/peso fresco do fruto; por outro lado, os tratamentos com PL-0D e PL-25D, os menos intensos - e de maior produtividade - por valores mais elevados de produtividade/gema, produtividade/ ha e produtividade/peso de ramos podados.

O teor de açúcar da uva - normalmente expresso em sólidos solúveis totais $\left({ }^{\circ} \mathrm{Brix}\right)$ e densidade - geralmente apresenta correlação negativa com a produtividade do vinhedo (PALLIOTTI; CARTECHINI, 2000; MORINAGA et al., 2003; CUS et al., 2004; DAMI et al., 2005; DAMI et al., 2006; VALDÉS et al., 2009; PASTORE et al., 2011; SANTESTEBAN et al., 2011; SUN et al., 2012). Entretanto, nem sempre a videira responde dessa forma, pois há trabalhos que mostram que aumentos na produtividade do vinhedo não causam efeitos consistentes no teor de açúcar da uva ou mesmo não proporcionam aumentos no ${ }^{\circ}$ Brix (KELLER et al., 2005; MATTII; FERRINI, 2005; IANNINI et al., 2007; REYNOLDS et al., 2007). Quanto à acidez, a literatura registra valores que indicam que há correlação negativa entre produtividade do vinhedo e $\mathrm{pH}$ (EZZAHOUANI; WILLIAMS, 2001; DAMI et al., 2006; SUN et al., 2012), o que também foi constatado neste trabalho. Mas há também quem mostre não ter havido correlação significativa entre essas variáveis (KELLER et al., 2005). Esses resultados evidenciam que, dependendo de determinadas condições, o efeito significativo do manejo do dossel vegetativo do vinhedo sobre variáveis relacionadas a componentes de produção da videira pode não ser constatado na composição físico-química do mosto da uva, pelo menos no que se diz respeito a variáveis relacionadas a açúcar e acidez. Ou seja, dentro de determinados limites, a planta exerce um poder autorregulador entre as partes vegetativa-produtiva e fisiológica-bioquímica.

Entretanto, tem-se que enfatizar que os vinhos de melhor qualidade, provenientes das mais importantes regiões vitícolas, são elaborados com uvas produzidas em vinhedos com produtividades medianas, especialmente em videiras conduzidas em espaldeira, que é o sistema de condução mais adotado ao redor do mundo. Em princípio, isso também ocorre em videiras conduzidas em latada, como é o caso das regiões vitícolas do Sul do Brasil. Este sistema de condução permite maior expansão do dossel vegetativo, o que, em geral, proporciona maior vigor da planta e maior produtividade do vinhedo. Isso, aliado às condições climáticas da região, que proporciona calor e umidade, pode aumentar a capacidade da planta em produzir maior quantidade de uva sem uma proporcional redução do teor de açúcar. Ressalte-se, entretanto, que o teor de açúcar da uva é um importante indicador de seu estado de maturação, mas não necessariamente da presença de substâncias que tenham efeito marcante, direta ou indiretamente, em sua qualidade, como as substâncias aromáticas, no caso de aromas primários do vinho, e os compostos fenólicos. Ou seja, uvas com as mesmas porcentagens de açúcar, provenientes de uma mesma região vitícola, podem originar vinhos com composição distinta e características sensoriais diferenciadas. 
TABELA 1 - Efeito das intensidades da poda seca e do desbaste de cacho na produtividade da gema e do vinhedo da cv. Cabernet Sauvignon.

\begin{tabular}{|c|c|c|c|c|c|c|c|c|}
\hline \multirow{2}{*}{ Tratamento $^{1}$} & \multicolumn{4}{|c|}{ Produtividade (g/gema) } & \multicolumn{4}{|c|}{ Produtividade $(\mathrm{kg} / \mathrm{ha})$} \\
\hline & $1^{\circ}$ ano & $2^{\circ}$ ano & $3^{\circ}$ ano & $4^{\circ}$ ano & $1^{\circ}$ ano & $2^{\circ}$ ano & $3^{\circ}$ ano & $4^{\circ}$ ano \\
\hline PC-0D & $151 \mathrm{a}^{2}$ & 79 a & $186 \mathrm{a}$ & $100 \mathrm{ab}$ & $21.007 \mathrm{bc}$ & $28.890 \mathrm{abc}$ & $24.838 \mathrm{abc}$ & $16.632 \mathrm{bc}$ \\
\hline PC-25D & $122 \mathrm{bc}$ & $144 \mathrm{abc}$ & $193 \mathrm{a}$ & $88 \mathrm{abc}$ & $17.567 \mathrm{c}$ & $24.926 \mathrm{bc}$ & $25.245 \mathrm{abc}$ & $15.634 \mathrm{bcd}$ \\
\hline PC-50D & $105 \mathrm{c}$ & $114 \mathrm{~cd}$ & $139 \mathrm{ab}$ & $69 \mathrm{bcd}$ & $14.785 \mathrm{~cd}$ & $19.323 \mathrm{~cd}$ & $19.738 \mathrm{bcd}$ & $11.933 \mathrm{~cd}$ \\
\hline PC-75D & $63 \mathrm{~d}$ & $86 \mathrm{~d}$ & $100 \mathrm{~b}$ & $42 \mathrm{~d}$ & 8.3 & 14. & 13. & 7.7 \\
\hline PL-0D & $158 \mathrm{a}$ & $161 \mathrm{ab}$ & $186 \mathrm{a}$ & $115 \mathrm{a}$ & $35.250 \mathrm{a}$ & $38.689 \mathrm{a}$ & $32.435 \mathrm{a}$ & $24.900 \mathrm{a}$ \\
\hline PL-25D & $128 \mathrm{~b}$ & $127 \mathrm{bcd}$ & $147 \mathrm{ab}$ & $92 \mathrm{abc}$ & $27.700 \mathrm{~b}$ & $29.664 \mathrm{ab}$ & $28.624 \mathrm{ab}$ & $21.554 \mathrm{ab}$ \\
\hline PL-50D & $107 \mathrm{c}$ & $117 \mathrm{~cd}$ & $141 \mathrm{ab}$ & 79 abcd & $24.589 \mathrm{~b}$ & $29.597 \mathrm{ab}$ & $26.543 \mathrm{abc}$ & $18.991 \mathrm{abc}$ \\
\hline PL-75D & $70 \mathrm{~d}$ & $96 \mathrm{~d}$ & $99 \mathrm{~b}$ & $56 \mathrm{~cd}$ & $14.056 \mathrm{~cd}$ & $23.375 \mathrm{bcd}$ & $19.227 \mathrm{~cd}$ & $13.170 \mathrm{~cd}$ \\
\hline $\mathrm{CV}(\%)$ & 8,26 & 15,79 & 21,66 & 23,12 & 16,49 & 18,23 & 19,00 & 23,52 \\
\hline ob. $>F^{3}$ & 0001 & 0,00001 & 0,00023 & 0,00007 & 0,00001 & 0,00001 & 0,00006 & 0,00002 \\
\hline
\end{tabular}

${ }^{1} \mathrm{PC}$ e $\mathrm{PL}=$ podas curta e longa; 0D, 25D, 50D e 75D = desbaste de cacho (\%).

${ }^{2}$ Médias seguidas da mesma letra, na coluna, não diferem entre si, pelo teste de Tukey, a 5\% de probabilidade.

${ }^{3}$ Resultado do teste $\mathrm{F}$ da variação entre tratamentos.

TABELA 2 - Efeito das intensidades da poda seca e do desbaste de cacho no peso de ramos podados da cv. Cabernet Sauvignon.

\begin{tabular}{|c|c|c|c|c|c|c|c|c|}
\hline \multirow{2}{*}{ Tratamento $^{1}$} & \multicolumn{4}{|c|}{ Peso de ramos podados (g/gema) } & \multicolumn{4}{|c|}{ Peso de ramos podados $(\mathrm{kg} / \mathrm{ha})$} \\
\hline & $1^{\circ}$ ano & $2^{\circ}$ ano & $3^{\circ}$ ano & $4^{\circ}$ ano & $1^{\circ}$ ano & $2^{\circ}$ ano & $3^{\circ}$ ano & $4^{\circ}$ ano \\
\hline PC-0D & $47 \mathrm{ab}^{2}$ & $37 \mathrm{ab}$ & $43 \mathrm{ab}$ & $32 \mathrm{ab}$ & $6.271 \mathrm{ab}$ & $5.492 \mathrm{a}$ & $5.607 \mathrm{a}$ & $5.055 \mathrm{a}$ \\
\hline PC-25D & $43 \mathrm{abc}$ & $29 \mathrm{abc}$ & $39 \mathrm{ab}$ & $36 \mathrm{ab}$ & $6.164 \mathrm{ab}$ & $4.451 \mathrm{a}$ & $5.441 \mathrm{a}$ & $6.013 \mathrm{a}$ \\
\hline PC-50D & $51 \mathrm{ab}$ & $38 \mathrm{a}$ & $49 \mathrm{a}$ & $38 \mathrm{a}$ & $7.108 \mathrm{a}$ & $6.134 \mathrm{a}$ & $6.783 \mathrm{a}$ & $6.521 \mathrm{a}$ \\
\hline PC-75D & $53 \mathrm{a}$ & $38 \mathrm{a}$ & $46 \mathrm{a}$ & $35 \mathrm{ab}$ & $6.713 \mathrm{ab}$ & $5.744 \mathrm{a}$ & $5.643 \mathrm{a}$ & $5.982 \mathrm{a}$ \\
\hline PL-0D & $20 \mathrm{~d}$ & $16 \mathrm{c}$ & $23 \mathrm{~b}$ & $23 \mathrm{~b}$ & $4.373 \mathrm{~b}$ & $3.640 \mathrm{a}$ & $4.245 \mathrm{a}$ & $4.779 \mathrm{a}$ \\
\hline PL-25D & $22 \mathrm{~d}$ & $16 \mathrm{c}$ & $23 \mathrm{~b}$ & $20 \mathrm{~b}$ & $4.497 \mathrm{ab}$ & $3.506 \mathrm{a}$ & $4.197 \mathrm{a}$ & $4.463 \mathrm{a}$ \\
\hline PL-50D & $27 \mathrm{~cd}$ & $19 \mathrm{bc}$ & $33 \mathrm{ab}$ & $27 \mathrm{ab}$ & $5.968 \mathrm{ab}$ & $4.852 \mathrm{a}$ & $6.335 \mathrm{a}$ & $6.087 \mathrm{a}$ \\
\hline PL-75D & $33 \mathrm{bcd}$ & $24 \mathrm{abc}$ & $32 \mathrm{ab}$ & $25 \mathrm{ab}$ & $6.823 \mathrm{ab}$ & $5.660 \mathrm{a}$ & $6.496 \mathrm{a}$ & $5.652 \mathrm{a}$ \\
\hline $\mathrm{CV}(\%)$ & 23,85 & 31,10 & 27,16 & 25,80 & 20,44 & 30,00 & 29,28 & 26,27 \\
\hline Prob. $>\mathrm{F}^{3}$ & 0,00001 & 0,00030 & 0,00107 & 0,00450 & 0,00954 & 0,07914 & 0,18685 & 0,35546 \\
\hline
\end{tabular}

${ }^{1} \mathrm{PC}$ e $\mathrm{PL}=$ podas curta e longa; $0 \mathrm{D}, 25 \mathrm{D}, 50 \mathrm{D}$ e $75 \mathrm{D}=$ desbaste de cacho $(\%)$.

${ }^{2}$ Médias seguidas da mesma letra, na coluna, não diferem entre si, pelo teste de Tukey, a 5\% de probabilidade.

${ }^{3}$ Resultado do teste $\mathrm{F}$ da variação entre tratamentos. 
TABELA 3 - Efeito das intensidades da poda seca e do desbaste de cacho nas relações área foliar/peso fresco do fruto e produtividade do vinhedo/peso de ramos podados da cv. Cabernet Sauvignon.

\begin{tabular}{|c|c|c|c|c|c|c|c|c|}
\hline \multirow[t]{2}{*}{ Tratamento $^{1}$} & \multicolumn{4}{|c|}{ Área foliar/Peso fresco do fruto $\left(\mathrm{cm}^{2} / \mathrm{g}\right)$} & \multicolumn{4}{|c|}{$\begin{array}{l}\text { Produtividade } / \text { Peso de ramos podados } \\
\qquad(\mathrm{kg} / \mathrm{kg})\end{array}$} \\
\hline & $1^{\circ}$ ano & $2^{\circ}$ ano & $3^{\circ}$ ano & $4^{\circ}$ ano & $1^{\circ}$ ano & $2^{\circ}$ ano & $3^{\circ}$ ano & $4^{\circ}$ ano \\
\hline PC-0D & $21,82 b^{2}$ & $22,55 \mathrm{~d}$ & $16,70 \mathrm{bc}$ & $26,93 \mathrm{bc}$ & $3,35 \mathrm{~cd}$ & $5,26 \mathrm{bcd}$ & $4,43 \mathrm{bc}$ & $3,29 \mathrm{ab}$ \\
\hline PC-25D & $16,52 \mathrm{bc}$ & $24,69 \mathrm{~cd}$ & $19,10 \mathrm{bc}$ & $34,99 \mathrm{bc}$ & 2,85 cde & $5,60 \mathrm{bcd}$ & $4,64 \mathrm{~b}$ & $2,60 \mathrm{~b}$ \\
\hline PC-50D & $27,10 \mathrm{~b}$ & $35,38 \mathrm{bc}$ & $22,94 \mathrm{abc}$ & $38,32 \mathrm{bc}$ & $2,08 \mathrm{de}$ & $3,15 \mathrm{~cd}$ & $2,91 \mathrm{bc}$ & $1,83 \mathrm{~b}$ \\
\hline PC-75D & $48,04 \mathrm{a}$ & $48,28 \mathrm{a}$ & $30,83 \mathrm{ab}$ & $64,44 \mathrm{a}$ & $1,24 \mathrm{e}$ & $2,44 \mathrm{~d}$ & $2,45 \mathrm{c}$ & $1,29 \mathrm{~b}$ \\
\hline PL-0D & $9,08 \mathrm{c}$ & $22,63 \mathrm{~d}$ & $16,38 \mathrm{bc}$ & $23,03 \mathrm{c}$ & $8,06 \mathrm{a}$ & $10,63 \mathrm{a}$ & $7,64 \mathrm{a}$ & $5,21 \mathrm{a}$ \\
\hline PL-25D & $15,42 \mathrm{bc}$ & $27,15 \mathrm{~cd}$ & $15,70 \mathrm{c}$ & $24,95 \mathrm{bc}$ & $6,16 \mathrm{ab}$ & $8,46 \mathrm{ab}$ & $6,82 \mathrm{a}$ & $4,83 \mathrm{a}$ \\
\hline PL-50D & $15,14 \mathrm{bc}$ & $32,93 \mathrm{bcd}$ & $21,03 \mathrm{abc}$ & $30,42 \mathrm{bc}$ & $4,12 \mathrm{c}$ & $6,10 \mathrm{bc}$ & $4,19 \mathrm{bc}$ & $3,12 \mathrm{ab}$ \\
\hline PL-75D & $30,94 \mathrm{ab}$ & $41,80 \mathrm{ab}$ & $34,61 \mathrm{a}$ & $44,88 \mathrm{ab}$ & $2,06 \mathrm{de}$ & $4,13 \mathrm{~cd}$ & $2,96 \mathrm{bc}$ & $2,33 \mathrm{~b}$ \\
\hline $\mathrm{CV}(\%)$ & 36,32 & 17,79 & 32,61 & 27,40 & 22,74 & 27,02 & 21,49 & 34,56 \\
\hline Prob. $>\mathrm{F}^{3}$ & 0,00002 & 0,00001 & 0,00159 & 0,00003 & 0,00001 & 0,00001 & 0,00001 & 0,00006 \\
\hline
\end{tabular}

${ }^{1} \mathrm{PC}$ e $\mathrm{PL}=$ podas curta e longa; $0 \mathrm{D}, 25 \mathrm{D}, 50 \mathrm{D}$ e $75 \mathrm{D}=$ desbaste de cacho $(\%)$.

${ }^{2}$ Médias seguidas da mesma letra, na coluna, não diferem entre si, pelo teste de Tukey, a 5\% de probabilidade.

${ }^{3}$ Resultado do teste $\mathrm{F}$ da variação entre tratamentos.

TABELA 4 - Efeito das intensidades da poda seca e do desbaste de cacho no ${ }^{\circ}$ Brix e na densidade do mosto da uva Cabernet Sauvignon.

\begin{tabular}{|c|c|c|c|c|c|c|c|c|}
\hline \multirow{2}{*}{ Tratamento $^{1}$} & \multicolumn{4}{|c|}{${ }^{\circ}$ Brix } & \multicolumn{4}{|c|}{ Densidade $(\mathrm{g} / \mathrm{mL})$} \\
\hline & $1^{\circ}$ ano & $2^{\circ}$ ano & $3^{\circ}$ ano & $4^{\circ}$ ano & $1^{\circ}$ ano & $2^{\circ}$ ano & $3^{\circ}$ ano & $4^{\circ}$ ano \\
\hline PC-0D & $20,8 \mathrm{a}^{2}$ & $19,9 \mathrm{a}$ & $18,6 \mathrm{a}$ & $19,4 \mathrm{a}$ & $1,0904 \mathrm{ab}$ & $1,0865 \mathrm{a}$ & $1,0813 \mathrm{a}$ & $1,0838 \mathrm{a}$ \\
\hline PC-25D & $21,5 \mathrm{a}$ & $19,5 a b$ & $18,4 \mathrm{a}$ & $19,3 \mathrm{a}$ & $1,0912 \mathrm{ab}$ & $1,0862 \mathrm{ab}$ & $1,0800 \mathrm{a}$ & $1,0834 \mathrm{a}$ \\
\hline PC-50D & $20,8 \mathrm{a}$ & $19,3 \mathrm{ab}$ & $18,2 \mathrm{a}$ & $19,1 \mathrm{a}$ & $1,0877 \mathrm{ab}$ & $1,0841 \mathrm{ab}$ & $1,0793 \mathrm{a}$ & $1,0820 \mathrm{a}$ \\
\hline PC-75D & $21,7 \mathrm{a}$ & $19,4 a b$ & $18,4 \mathrm{a}$ & $19,5 \mathrm{a}$ & 1,0918 a & $1,0850 \mathrm{ab}$ & $1,0801 \mathrm{a}$ & $1,0842 \mathrm{a}$ \\
\hline PL-0D & $20,4 \mathrm{a}$ & $18,9 \mathrm{~b}$ & $18,3 \mathrm{a}$ & $19,5 \mathrm{a}$ & $1,0863 \mathrm{~b}$ & $1,0821 \mathrm{~b}$ & $1,0795 \mathrm{a}$ & $1,0839 \mathrm{a}$ \\
\hline PL-25D & $20,4 \mathrm{a}$ & $19,3 \mathrm{ab}$ & $18,3 \mathrm{a}$ & $19,4 \mathrm{a}$ & $1,0866 \mathrm{ab}$ & $1,0840 \mathrm{ab}$ & $1,0795 \mathrm{a}$ & $1,0835 \mathrm{a}$ \\
\hline PL-50D & $20,7 \mathrm{a}$ & $19,6 \mathrm{ab}$ & $18,3 \mathrm{a}$ & $19,9 \mathrm{a}$ & $1,0877 \mathrm{ab}$ & $1,0855 \mathrm{ab}$ & 1,0799 a & $1,0856 \mathrm{a}$ \\
\hline PL-75D & $21,6 \mathrm{a}$ & $19,7 \mathrm{a}$ & $18,5 \mathrm{a}$ & $19,9 \mathrm{a}$ & $1,0912 \mathrm{ab}$ & $1,0862 \mathrm{a}$ & $1,0806 \mathrm{a}$ & $1,0860 \mathrm{a}$ \\
\hline $\mathrm{CV}(\%)$ & 3,48 & 1,92 & 2,70 & 4,32 & 0,25 & 0,16 & 0,19 & 0,34 \\
\hline Prob. $>\mathrm{F}^{3}$ & 0,03139 & 0,01002 & 0,88993 & 0,77203 & 0,00652 & 0,01213 & 0,83631 & 0,76976 \\
\hline
\end{tabular}

${ }^{\mathrm{T}} \mathrm{PC}$ e $\mathrm{PL}=$ podas curta e longa; 0D, 25D, 50D e 75D = desbaste de cacho (\%).

${ }^{2}$ Médias seguidas da mesma letra, na coluna, não diferem entre si, pelo teste de Tukey, a 5\% de probabilidade.

${ }^{3}$ Resultado do teste $\mathrm{F}$ da variação entre tratamentos. 
TABELA 5 - Efeito das intensidades da poda seca e do desbaste de cacho na acidez titulável e no pH do mosto da uva Cabernet Sauvignon.

\begin{tabular}{|c|c|c|c|c|c|c|c|c|}
\hline \multirow{2}{*}{ Tratamento $^{1}$} & \multicolumn{4}{|c|}{ Acidez titulável (g ácido tartárico/100 mL) } & \multicolumn{4}{|c|}{$\mathrm{pH}$} \\
\hline & $1^{\circ}$ ano & $2^{\circ}$ ano & $3^{\circ}$ ano & $4^{\circ}$ ano & $1^{\circ}$ ano & $2^{\circ}$ ano & $3^{\circ}$ ano & $4^{\circ}$ ano \\
\hline PC-0D & $0,87 a b^{2}$ & $0,98 \mathrm{a}$ & $1,14 \mathrm{a}$ & $1,19 \mathrm{a}$ & $3,26 \mathrm{ab}$ & $3,28 a b$ & $3,30 \mathrm{a}$ & $3,27 \mathrm{ab}$ \\
\hline PC-25D & $0,83 \mathrm{ab}$ & $1,04 \mathrm{a}$ & $1,15 \mathrm{a}$ & $1,22 \mathrm{a}$ & $3,30 \mathrm{ab}$ & $3,34 \mathrm{a}$ & $3,32 \mathrm{a}$ & $3,29 \mathrm{ab}$ \\
\hline PC-50D & $0,91 \mathrm{a}$ & $1,03 \mathrm{a}$ & $1,17 \mathrm{a}$ & $1,18 \mathrm{a}$ & $3,25 \mathrm{ab}$ & $3,30 \mathrm{a}$ & $3,31 \mathrm{a}$ & $3,30 \mathrm{ab}$ \\
\hline PC-75D & $0,92 \mathrm{a}$ & $1,03 \mathrm{a}$ & $1,13 \mathrm{a}$ & $1,14 \mathrm{a}$ & $3,33 \mathrm{a}$ & $3,33 \mathrm{a}$ & $3,32 \mathrm{a}$ & $3,32 \mathrm{a}$ \\
\hline PL-0D & $0,81 \mathrm{~b}$ & $0,99 \mathrm{a}$ & $1,11 \mathrm{a}$ & $1,14 \mathrm{a}$ & $3,23 \mathrm{~b}$ & $3,21 \mathrm{~b}$ & $3,28 \mathrm{a}$ & $3,23 \mathrm{a}$ \\
\hline PL-25D & $0,89 \mathrm{ab}$ & $1,00 \mathrm{a}$ & $1,16 \mathrm{a}$ & $1,18 \mathrm{a}$ & $3,22 \mathrm{~b}$ & $3,27 a b$ & $3,30 \mathrm{a}$ & $3,25 \mathrm{ab}$ \\
\hline PL-50D & $0,89 \mathrm{ab}$ & $1,04 \mathrm{a}$ & $1,11 \mathrm{a}$ & $1,16 \mathrm{a}$ & $3,26 \mathrm{ab}$ & $3,30 \mathrm{a}$ & $3,30 \mathrm{a}$ & $3,31 \mathrm{a}$ \\
\hline PL-75D & $0,88 \mathrm{ab}$ & $1,01 \mathrm{a}$ & $1,08 \mathrm{a}$ & $1,14 \mathrm{a}$ & $3,33 \mathrm{a}$ & $3,34 \mathrm{a}$ & $3,32 \mathrm{a}$ & $3,31 \mathrm{a}$ \\
\hline $\mathrm{CV}(\%)$ & 5,63 & 6,75 & 10,72 & 4,54 & 1,25 & 1,12 & 1,38 & 1,04 \\
\hline Prob. $>\mathrm{F}^{3}$ & 0,01683 & 0,79287 & 0,95994 & 0,21513 & 0,00099 & 0,00059 & 0,81819 & 0,00481 \\
\hline
\end{tabular}

${ }^{1} \mathrm{PC}$ e $\mathrm{PL}=$ podas curta e longa; $0 \mathrm{D}, 25 \mathrm{D}, 50 \mathrm{D}$ e $75 \mathrm{D}=$ desbaste de cacho $(\%)$.

${ }^{2}$ Médias seguidas da mesma letra, na coluna, não diferem entre si, pelo teste de Tukey, a $5 \%$ de probabilidade.

${ }^{3}$ Resultado do teste $\mathrm{F}$ da variação entre tratamentos.
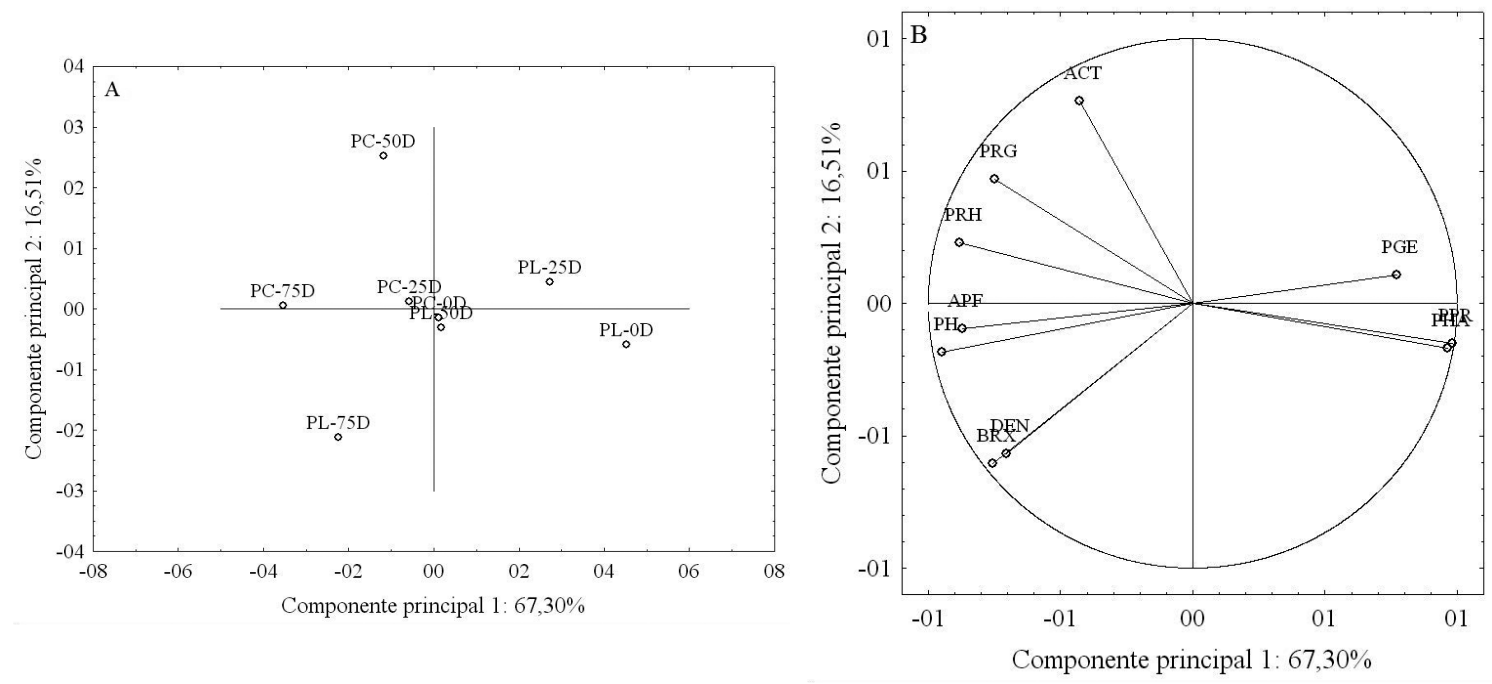

FIGURA 1 - Projeção dos tratamentos (A) e das variáveis (B) nos planos formados pelos componentes principais $1 \times 2$. Legenda $(A): \mathrm{PC}=$ poda curta; $\mathrm{PL}=$ poda longa; $0 \mathrm{D}, 25 \mathrm{D}, 50 \mathrm{D}, 75 \mathrm{D}=$ porcentagem de desbaste de cacho. Legenda (B): $\mathrm{PGE}=$ produtividade $(\mathrm{g} / \mathrm{gema}), \mathrm{PHA}=$ produtividade $(\mathrm{kg} / \mathrm{ha})$, $\mathrm{PRG}=$ peso de ramos podados $(\mathrm{g} / \mathrm{gema}), \mathrm{PRH}=$ peso de ramos podados $(\mathrm{kg} / \mathrm{ha}), \mathrm{APF}=$ área foliar/ peso fresco do fruto, $\mathrm{PPR}=$ produtividade/peso de ramos podados $(\mathrm{kg} / \mathrm{kg}), \mathrm{BRX}={ }^{\circ} \mathrm{Brix}, \mathrm{DEN}=$ densidade $(\mathrm{g} / \mathrm{mL}), \mathrm{ACT}=$ acidez titulável ( $\mathrm{g}$ ácido tartárico $/ 100 \mathrm{~mL}), \mathrm{PH}=\mathrm{pH}$. 


\section{CONCLUSÕES}

1-Dois níveis de poda seca e quatro de desbaste de cacho têm efeito significativo na produtividade por gema e produtividade do vinhedo; no peso de ramos podados por gema e por hectare; e nas relações área foliar/peso fresco do fruto e produtividade/peso de ramos podados da videira Cabernet Sauvignon.

2-Os tratamentos poda curta e poda longa, com $75 \%$ de desbaste de cacho, são os que, em geral, apresentam as menores produtividades por hectare $\mathrm{e}$ maior relação área foliar/peso fresco do fruto.

3 -Os tratamentos poda longa com $0 \%$ e $25 \%$ de desbaste de cacho proporcionam maior relação produtividade/peso de ramos podados.

4-Os tratamentos de poda seca e de desbaste de cacho não têm efeito expressivo sobre as variáveis relacionadas a açúcar $-{ }^{\circ}$ Brix e densidade - e acidez - pH e acidez titulável - do mosto da uva Cabernet Sauvignon.

5-A análise de componentes principais discrimina principalmente o tratamento de poda curta com $75 \%$ de desbaste, que é o mais intenso e de menor produtividade, de poda longa a $0 \%$ e $25 \%$ de desbaste de cacho, os menos intensos e de maior produtividade.

\section{REFERÊNCIAS}

BERKEY, T. G.; MANSFIELD, A. K.; LERCH, S. D.; MEYERS, J. M.; HEUVEL, J. E. V. Crop load adjustment in 'Seyval Blanc' winegrape: impacts on yield components, fruit composition, consumer wine preferences, and economics of production. HortTechnology, Alexandria, v. 21, p. 593-598, 2011.

BRAVDO, B.; HEPNER, Y; LOINGER, C.; COHEN, S.; TABACMAN, H. Effect of crop level and crop load on growth, yield, must and wine composition, and quality of Cabernet Sauvignon. American Journal of Enology and Viticulture, Davis, v. 36, p. 125-131, 1985.

BRAVDO, B.; HEPNER, Y.; LOINGER, C.; COHEN, S.; TABACMAN, H. Effect of crop level on growth, yield and wine quality of a high yielding Carignane vineyard. American Journal of Enology and Viticulture, Davis, v. 35, p. 247-252, 1984.
BUBOLA, M.; PERSURIC, D.; GANIC, K. K. Impact of cluster thinning on productive characteristics and wine phenolic composition of cv. Merlot. Journal of Food, Agriculture \& Environment, Helsinki, v. 9, p. 36-39, 2011

CUS, F.; KORUZA, Z. K.; KORUZO, B.; LAVRENCIC, P. Influence of crop load and cultivar's dependent canopy management on grape must quality of 'Sipon', 'Zametovka' and 'Rebula'. Acta Horticulturae, The Hague, n. 652, p. 141-146, 2004.

DAMI, I.; FERREE, D. C.; KURTURAL, S. K.; TAYLOR, B. H. Influence of crop load on 'Chambourcin' yield, fruit quality, and winter hardiness under Midwestern United States environmental conditions. Acta Horticulturae, The Hague, n. 689, p. 203-208, 2005.

DAMI, I.; FERREE, D.; PRAJITNA，A.; SCURLOCK, D. A five-year study on the effect of cluster thinning on yield and fruit composition of 'Chambourcin' grapevines. HortScience, Alexandria, v. 41, p. 586-588, 2006.

EZZAHOUANI, A.; WILLIAMS, L. E. The effects of thinning and girdling on leaf water potential, growth and fruit composition of Ruby Seedless grapevines. Journal International des Sciences de la Vigne et du Vin, Bordeaux, v. 35, p. 79-85, 2001.

GIL-MUÑOZ，R. R.; VILA-LÓPEZ，J. I.; FERNÁNDEZ-FERNÁNDEZ; MARTÍNEZCUTILLAS, A. Effects of cluster thinning on anthocyanin extractability and chromatic parameters of Syrah and Tempranillo grapes and wines. Journal International des Sciences de la Vigne et du Vin, Bordeaux, v. 43, p. 45-53, 2009.

IANNINI, C.; MATTII, G. B.; RIVELLI, A. R.; ROTUNDO, A. Leaf removal and cluster thinning trials in Aglianico grapevine. Acta Horticulturae, The Hague, n. 754, p. 241-247, 2007.

JACKSON, D. I. Factors affecting soluble solids, acid, $\mathrm{pH}$, and color in grapes. American Journal of Enology and Viticulture, Davis, v.37, p.179183,1986

JACKSON, D. I.; LOMBARD, P. B. Environmental and management practices affecting grape composition and wine quality - A review. American Journal of Enology and Viticulture, Davis, v. 44, p. 409-430, 1993. 
KAPS, M. L.; CAHOON, G. A. Growth and fruiting of container-grown Seyval blanc grapevines modified by changes in crop level, leaf number and position, and light exposure. American Journal of Enology and Viticulture, Davis, v. 43, p. 191-199, 1992.

KELLER, M.; MILLS, L. J.; WAMPLE, R. L.; SPAYD, S. E. Cluster thinning effects on three deficit-irrigated Vitis vinifera cultivars. American Journal of Enology and Viticulture, Davis, v. 56, p. 91-103, 2005.

KELLER, M.; SMITHYMAN, R. P.; MILLS, L. J. Interactive effects of deficit irrigation and crop load on Cabernet Sauvignon in an arid climate. American Journal of Enology and Viticulture, Davis, v. 59, p. 221-234, 2008.

KLIEWER, W. M.; ANTCLIFF, A. J. Influence of defoliation, leaf darkening, and cluster shading on the growth and composition of Sultana grapes. American Journal of Enology and Viticulture, Davis, v. 21, p. 26-36, 1970.

KLIEWER, W. M.; WEAVER, R. J. Effect of crop level and leaf area on growth, composition, and coloration of 'Tokay' grapes. American Journal of Enology and Viticulture, Davis, v. 22, p. 172-177, 1971.

KOK, D. Influences of pre-and post-veraison cluster thinning treatments on grape composition variables and monoterpene levels of Vitis vinifera L. cv. Sauvignon Blanc. Journal of Food, Agriculture \& Environment, Helsinki, v. 9, p. 22-26, 2011.

MANDELLI, F.; MIELE, A. Manejo do dossel vegetativo e seu efeito nos componentes de produção da videira Merlot. Revista Brasileira de Fruticultura, Jaboticabal, v. 34, n. 4, p. 964-973, 2012.

MATTII, G. B.; FERRINI, F. The effects of crop load on 'Sangiovese' grapevines. Acta Horticulturae, The Hague, n. 689, p. 239-242, 2005.

MORINAGA, K.; IMAI, S.; YAKUSHIJI, H.; KOSHITA, Y. Effects of fruit load on partitioning of ${ }^{15} \mathrm{~N}$ and ${ }^{13} \mathrm{C}$, respiration, and growth of grapevine roots at different fruit stages. Scientia Horticulturae, Amsterdam, v. 97, p. 239-253, 2003.
MURISIER, F.; ZIEGLER, R. Effets de la charge en bourgeons et de la densité de plantation sur le potentiel de production, sur la qualité du raisin et sur le développement végétatif. Revue Suisse de Viticulture, Arboriculture, Horticulture, Nyon, v. 23, p. 277-282, 1991.

NAOR, A.; GAL, Y.; BRAVDO, B. Shoot and cluster thinning influence vegetative growth, fruit yield, and wine quality of 'Sauvignon blanc' grapevines. Journal of the American Society for Horticultural Science, Mont Vernon, v. 127, p. 628-634, 2002.

PALLIOTTI, A.; CARTECHINI, A. Cluster thinning effects on yield and grape composition in different grapevine cultivars. Acta Horticulturae, The Hague, n. 512, p. 111-119, 2000.

PASTORE, C.; ZENONI, S.; TORNIELLI, G. B.; ALLEGRO, G.; DAL SANTO, S.; VALENTINI, G.; INTRIERI, C.; PEZZOTTI, M.; FILIPPETTI, I. Increasing the source/sink ratio in Vitis vinifera (cv Sangiovese) induces extensive transcriptome reprogramming and modifies berry ripening. BMC Genomics, Nova York, v. 12, ar. 631, 2011.

REYNOLDS, A. G.; SCHLOSSER, J.; SOROKOWSKY, D.; ROBERTS, R.; WILLWERTH, J.; SAVIGNY, C. de. Magnitude of viticultural and enological effects. II. Relative impacts of cluster thinning and yeast strain on composition and sensory attributes of Chardonnay Musqué. American Journal of Enology and Viticulture, Davis, v. 58, p. 25-41, 2007.

RIBÉREAU-GAYON, J.; PEYNAUD, E.; SUDRAUD, P.; RIBÉREAU-GAYON, P. Traité d'Enologie: sciences et techniques du vin: analyse et contrôle des vins. $2^{\text {nd }}$ ed. Paris: Dunod, 1982. v. $1,645 \mathrm{p}$.

SANTESTEBAN, L. G.; MIRANDA, C.; ROYO, J. B. Thinning intensity and water regime affect the impact cluster thinning has on grape quality. Vitis, Geilweilerhof, v.50, p.159-165, 2011.

SMART, R. E. Principles of grapevine canopy microclimate manipulation with implications for yield and quality. A review. American Journal of Enology and Viticulture, Davis, v. 36, p. 230-239, 1985. 
SUN, Q.; SACKS, G. L.; LERCH, S. D.; HEUVEL, J. E. V. Impact of shoot and cluster thinning on yield, fruit composition, and wine quality of Corot noir. American Journal of Enology and Viticulture, Davis, v. 63, p. 49-56, 2012.

SUN, Q.; SACKS, G. L.; LERCH, S. D.; HEUVEL, J. E. V. Impact of shoot thinning and harvest date on yield components, fruit composition and wine quality of Marechal Foch. American Journal of Enology and Viticulture, Davis, v. 62, p. 32-41, 2011.
VALDÉS, M. E.; MORENO, D.; GAMERO, E.; URIARTE, D.; PRIETO, M. del H.; MANZANO, R.; PICÓN, J.; INTRIGLIOLO, D. S. Effects of cluster thinning and irrigation amount on water relations, growth, yield and fruit and wine composition of Tempranillo grapes in Extremadura (Spain). Journal International des Sciences de la Vigne et du Vin, Bordeaux, v. 43, p. 67-76, 2009. 